\title{
RHO GTPASES SHOW DIFFERENTIAL SENSITIVITY TO \\ NUCLEOTIDE TRIPHOSPHATE DEPLETION IN A MODEL OF \\ ISCHEMIC CELL INJURY
}

\author{
Mark A. Hallett, Pierre C. Dagher and Simon J. Atkinson \\ Department of Medicine \\ Indiana University School of Medicine \\ Indianapolis, Indiana
}

Running Title: Rho Activity and Ischemia

Author for Correspondence:

Simon J. Atkinson, Ph.D.

Indiana University School of Medicine

Department of Medicine-Nephrology

950 W. Walnut St., R2-202

Indianapolis, IN 46202

Phone: (317) 278-0435

Fax: (317) 274-8575

Email: satkinso@iupui.edu 


\begin{abstract}
Rho GTPases are critical for actin cytoskeletal regulation, and alterations in their activity may contribute to altered cytoskeletal organization that characterizes many pathological conditions, including ischemia. G-protein activity is a function of the ratio of GTP-bound (active) to GDP-bound (inactive) protein, but the effect of altered energy metabolism on Rho protein activity has not been determined. We used antimycin A and substrate depletion to induce depletion of intracellular ATP and GTP in the kidney proximal tubule cell line LLC-PK 10 , and measured the activity of RhoA, Rac1 and Cdc42 using GTPase effector binding domains fused to glutathione S-transferase. RhoA activity decreased in parallel with the concentration of ATP and GTP during depletion so that by 60 minutes there was no detectable RhoA-GTP, and recovered rapidly when cells were returned to normal culture conditions. Dissociation of the membrane-actin linker ezrin, a target of RhoA signaling, from the cytoskeletal fraction paralleled the decrease in RhoA activity, and was augmented by treatment with the Rho kinase inhibitor Y27632. The activity of Cdc42 did not decrease significantly during depletion or recovery. Rac1 activity decreased moderately to a minimum at 30 minutes of depletion, but then increased from 30-90 minutes of depletion, even as ATP and GTP levels continued to fall. Our data are consistent with a principal role for RhoA in cytoskeletal reorganization during ischemia, and demonstrate that the activity of Rho GTPases can be maintained even at low GTP concentrations.
\end{abstract}

KEYWORDS: RAC, CDC42, ACTIN, EZRIN, ATP, GTP

Rho Activity and Ischemia 


\section{Introduction}

Rho family GTPases are members of the Ras superfamily of p21 G-proteins (25). They play a central role in regulating the organization and dynamics of the cytoskeleton, and thus integrate cell morphology and behavior with environmental signals and cellular physiology (6). The biological activities of small GTPases are regulated by their guanine nucleotide binding state (12). They interact with and activate their effectors when in the GTP-bound state and are inactive when the bound nucleotide is GDP, and in this way act as protein molecular switches. The position of the switch is set by the activity of antagonistic regulatory proteins, since the intrinsic GTPase rates and nucleotide dissociation rates for these proteins are slow. GTPase activating proteins (GAPs) stimulate nucleotide hydrolysis, thereby turning off the signal, whereas guanine nucleotide exchange factors (GEFs) stimulate GDP release and its replacement with GTP (the latter normally being the predominant guanine nucleotide in the cytoplasm) thus turning on signaling (7). However, these mechanisms, as they are commonly presumed to function, presuppose the nucleotide concentrations that prevail in normal healthy cells, but data are currently lacking on the effect of altered guanine nucleotide concentrations on p21 GTPase activity.

Ischemia is a pathophysiological state that is characterized in part by poor tissue perfusion and subsequent anoxia, with inhibition of oxidative phosphorylation due to substrate depletion (13). Although ischemia is a complex multi-factorial process, many of its consequences result from, and can be recapitulated by, depletion of the normal intracellular concentration of ATP (3). Ischemic injury to the epithelial cells of the kidney proximal tubule is a factor in the initiation and progression of acute renal failure, and 
typifies many of the features of ischemic injury in other cell types and organs. The normal architecture of the actin cytoskeleton is an early casualty of ischemic injury and ATP depletion, and this disruption directly results in impaired cell-cell and cell-substrate adhesion, loss of tight junction barrier function $(3,17)$ and mixing of apical and basolateral transporters (30), with resultant impaired organ physiology and structure. There is a loss of actin from the apical microvilli, terminal web, cortical junctional complexes and stress fibers. This results in membrane blebbing, internalization and loss of apical microvilli $(15,40)$. The loss of actin and villin from microvilli is coincident with abnormal perinuclear accumulation of F-actin (42) and an increase in the ratio of F- to Gactin in the cell.

The complexity and pervasiveness of the cytoskeletal alterations that are precipitated by ischemic injury indicate that they are probably the result of the combined effect of many different pathways. Perturbations of normal signaling pathways are probably a major factor in this aspect of injury, and in view of their critical role in cytoskeletal regulation, Rho GTPases are obvious candidate molecules. Evidence for the role of RhoA during ATP depletion in kidney epithelium comes from experiments utilizing the constitutively active Rho mutant, RhoV14. When RhoV14 is microinjected into tissue culture cells derived from renal proximal tubule epithelium before ATP depletion, cortical actin and stress fibers are maintained during subsequent depletion (33). Conversely, when the bacterial toxin C3 transferase, which specifically inactivates Rho, is injected stress fibers are not reassembled during recovery from ATP depletion. RhoV14 also protects tight junctions against disassembly during ATP depletion (17), 
while the corresponding constitutively active mutant of Rac, RacV12, protects adherens junctions (16).

Additional evidence for altered activity of Rho GTPases during ischemia or nucleotide triphosphate depletion is furnished by altered regulation of cytoskeletal proteins known to be downstream targets of Rho GTPase signaling. For example, ezrin is a protein link between the plasma membrane and the actin cytoskeleton (1) that is regulated by RhoA. In normal proximal tubule epithelial cells ezrin is localized in microvilli and at sites of cell-cell contact $(19,35)$. Ezrin binds to the cytoplasmic domain of the cell surface glycoprotein, CD44 (36) and the carboxy-terminus of ezrin binds to actin (37). The interaction of ezrin and CD44 is of high affinity only in the presence of phosphatidylinositol 4,5-bisphosphate (21). RhoA is thought to regulate this interaction through its effector, phosphatidylinositol 4-phosphate 5-kinase (29). Another effector of RhoA, Rho kinase (ROK), phosphorylates ezrin in vitro (28). The dephosphorylation of ezrin is an early event during ischemia in renal epithelium (8). As a result of dephosphorylation, ezrin dissociates from the cytoskeleton and can be extracted with non-ionic detergents.

The intracellular concentration of GTP is tightly coupled to that of ATP, and the two decrease in tandem with ischemia or chemical anoxia (10). Given the evidence for involvement of Rho GTPase signaling and the paucity of data concerning the effect of intracellular GTP levels on Rho protein signaling mechanisms in vivo, we used the ability of the GTPase binding domains from Rho effectors to discriminate between the GTP- and GDP-bound forms of the GTPase (24) to measure the relative amount of active and inactive RhoA, Rac1 and Cdc42 during ATP depletion induced by treatment with the 
mitochondrial poison antimycin A combined with substrate depletion. We measured ATP and GTP levels in parallel with these assays, to establish the degree of correlation between the activation state of the GTPase and nucleotide levels in the cell. We also followed the localization of actin stress fibers and the detergent solubility of ezrin, during nucleotide triphosphate depletion and recovery. Finally, we measured the activity of RhoA, Rac1 and Cdc42 during nucleotide depletion and recovery in the presence of constitutively active RhoAL63. 


\section{Materials and Methods}

Chemicals. All chemicals are from Sigma Chemical Co., St Louis, MO unless otherwise stated.

Cell culture. LLC-PK 10 porcine proximal tubule cells were grown with KP medium (1:1 DMEM/Ham's F-12 medium supplemented with 100 IU/ml penicillin, 100 $\mu \mathrm{g} / \mathrm{ml}$ streptomycin, $14 \mathrm{mM} \mathrm{NaHCO} 3$, and $12.5 \mathrm{mM}$ HEPES) with $10 \%$ fetal bovine serum in a humidified chamber $\left(95 \%\right.$ air- $\left.5 \% \mathrm{CO}_{2}\right)$ at $37^{\circ} \mathrm{C}$. Cells were grown in collagen coated T-25 flasks (Techno Plastic Products, Switzerland). Cells were grown two days post-confluence and rinsed with warm PBS before use. To model ischemia, cells were incubated with depleted DMEM (medium without amino acids, glucose, serum and antibiotics) and $100 \mathrm{nM}$ antimycin A. If recovery of these cells was required, they were rinsed with warm PBS and incubated with supplemented KP medium. For some experiments cells were rinsed twice and incubated with serum-free normal growth medium to control for the effect of removing growth factors. We analyzed the effect of ATP depletion in quiescent cells by incubating cells in serum-free medium for 24 hours prior to the experiment.

Nucleotide triphosphate assay. Cell cultures were rinsed three times with ice-cold PBS, scraped with $300 \mu 1$ of ice-cold acetonitrile followed by $700 \mu 1$ of ice-cold water (2) The extract was centrifuged at $16,000 \mathrm{~g}$ for 10 minutes at $4^{\circ} \mathrm{C}$. The supernatant was gassed with $\mathrm{N}_{2}$ while on ice for 30 minutes to evaporate the acetonitrile. The residue was solubilized with $1 \mathrm{~N} \mathrm{NaOH}$ and the protein concentration determined by Coomassie blue assay (Pierce Chemical, Rockford, IL). To separate nucleotide triphosphates, samples were diluted 1:1 and 100 $\mu$ l was injected onto a 4- $\mu$ m Nova-Pak C18 HPLC cartridge 
(100 X 8 mm I.D.) equipped with a radial compression chamber (Waters, Milford, MA). The elution buffer consisted of $20 \%$ acetonitrile, $10 \mathrm{mM}$ ammonium phosphate and $2 \mathrm{mM}$ PIC Reagent A ion-pairing reagent (Waters, Milford, MA). The column was run isocratically at $2 \mathrm{ml} / \mathrm{min}$ (18) on a Hewlett Packard Chemstation model 1100 with UV detection at 254nm (Hewlett-Packard, Wilmington, DE). Internal standards showed that nucleotide recovery was greater than $90 \%$.

Visualization of stress fibers. Cells were plated on collagen-coated coverslips in $35 \mathrm{~mm}$ Petri dishes. Cells were treated with antimycin A as previously described. After the depletion time course, coverslips were fixed in PBS / 5\% formaldehyde, permeabilized with PBS plus $0.2 \%$ Triton X-100 and incubated with $0.66 \mu \mathrm{M}$ rhodamine phalloidin (Molecular Probes, Eugene, OR). Confocal images were collected with a Zeiss Axiovert LSM510.

GTPase activity assay. The Rho kinase Rho binding domain (ROK-BD, amino acids 941-1075) and the Pak (p-21 associated kinase) binding domain (Pak-BD, amino acids 67-150) cloned into pGEX vectors were transformed into E. coli strain, BL21. pGEX-ROK-BD was a kind gift from Dr. Kozo Kaibuchi (Nagoya University, Japan) (27) and pGEX-Pak-BD was a kind gift from Dr. Gary Bokoch (Scripps Research Institute, La Jolla, CA) (4). Production of the fusion protein was initiated with isopropyl $\beta$-D-thioglactopyranoside (IPTG). The fusion protein was isolated by binding to Shexylglutathione-agarose as described in the manufacturers instructions. The glutathione S-transferase (GST) fusion proteins bound to glutathione agarose (GST-ROK-BD and GST-Pak-BD) were stored in aliquots in liquid nitrogen for no more than one month. The 
amount of fusion protein required to capture all of the available GTPase was determined empirically.

Affinity isolation was carried out as described (5) with modifications. After treatment with antimycin A cells were rinsed with warm PBS. $350 \mu$ l of buffer A [25mM Tris pH 7.5@ $4^{\circ} \mathrm{C}, 150 \mathrm{mM} \mathrm{K}$ acetate, 5mM EDTA, 5mM EGTA, 10mM dithiothreitol (DTT), $1 \%$ Triton X-100, 60mM n-octyl $\beta$-D-glucopyranoside (OG), 50 $\mu \mathrm{M}$ butylated hydroxy toluene (BHT), 1mM phenyl methyl sulfonyl fluoride (PMSF), 1mM benzamidine, $2 \mu \mathrm{g} / \mathrm{ml}$ aprotinin (Roche Molecular Biochemicals, Indianapolis, IN), $1 \mu \mathrm{g} / \mathrm{ml}$ Pepstatin A (Roche Molecular Biochemicals, Indianapolis, IN), 50 $\mu \mathrm{g} / \mathrm{ml}$ Leupeptin (Peptides International, Osaka, Japan), 40 $\mathrm{g} / \mathrm{ml}$ Bestatin (Roche Molecular Biochemicals, Indianapolis, IN)] was added to the T25 flasks and incubated on ice for 10 minutes with periodic manual rocking to evenly distribute the extraction buffer. The buffer was removed and centrifuged briefly at $15,000 \mathrm{~g} .25 \mu \mathrm{l}$ of detergent extract was mixed with $30 \mu 1$ of GST-ROK-BD to assay for RhoA activity, or $15 \mu 1$ of GST-Pak-BD to assay for Rac1 or $\mathrm{Cdc} 42$ activity, and incubated at $4^{\circ} \mathrm{C}$ for 1 hour on a rotator. The GST-binding domain was rinsed 3 times with $500 \mu l$ of buffer A without detergent or protease inhibitors. This unbound fraction was precipitated with $10 \%$ trichloroacetic acid, rinsed with $70 \%$ ethanol, acetone, dried and dissolved in SDS sample buffer (50mM Tris 7.5, 2\% sodium dodecyl sulfate (SDS), 5\% $\beta \mathrm{ME}$ (beta mercaptoethanol), $6 \mathrm{M}$ urea, bromophenol blue). The bound fraction was dried in a vacuum and solubilized in SDS sample buffer. Bound and unbound fractions for each time point were run on a $15 \%$ SDS PAGE polyacrylamide gel (Biowhittaker Molecular Applications, Rockland, ME). This was followed by western blotting and immunostaining with $1 \mu \mathrm{g} / \mathrm{ml}$ anti-RhoA antibody 
(26C4, Santa Cruz Biotechnology, Santa Cruz, CA), $2 \mu \mathrm{g} / \mathrm{ml}$ anti-Rac1 antibody (Santa Cruz Biotechnology, Santa Cruz, CA) or $2 \mu \mathrm{g} / \mathrm{ml}$ anti-Cdc42 antibody (Santa Cruz Biotechnology, Santa Cruz, CA) and horseradish peroxidase conjugated anti-mouse secondary antibody (Jackson Immunoresearch, West Grove, PA). Blots were visualized with ECL (Amersham Pharmacia Biotech, Piscataway, NJ) and Biomax ML film (Kodak, Rochester, NY). To determine the ratio of bound to unbound GTPase, film images were digitized and the optical density of each band was quantified using a Fluor-S MultiImager with Quantity One 4.1 software (Biorad Laboratories, Hercules, CA).

For assay validation experiments the detergent lysate was incubated with $1 \mathrm{mM}$ GDP, $1 \mathrm{mM} \mathrm{GTP} \gamma \mathrm{S}$ or without additional nucleotide for 10 minutes at $30^{\circ} \mathrm{C}$. As a control for non-specific binding to GST a sample of lysate was incubated with glutathioneagarose-GST.

To ensure the validity of using a ratio of bound to unbound GTPase to express activity, the levels of the GTPases were measured by densitometry of immunoblots of whole cell extracts and normalized to the total amount of protein in LLC-PK 10 cell lysates. When expressed as GTPase per $\mu \mathrm{g}$ of total protein for each of three time points, 0 and 90 minutes of nucleotide triphosphate depletion and 90 minutes followed by 60 minutes of repletion, there was no significant change in GTPase (RhoA, Rac1 and Cdc42) concentration during the time course of the experiment (data not shown).

Ezrin solubility assay. The solubility of ezrin during depletion and recovery was determined using the lysates from the RhoA activity assay. The amount of ezrin in the detergent insoluble fraction left after making the lysate was determined by adding SDS sample buffer to the T-flask containing the detergent insoluble adherent cell layer and 
heating it to $80^{\circ} \mathrm{C}$ for 2 minutes. The viscous solution was scraped out, sonicated to reduce the viscosity and run with the detergent soluble fractions on a $12 \%$ PAGE gel. Immunoblotting was performed using an anti-ezrin polyclonal antibody (kind gift of Jing Chen, Indiana State University, Terre Haute, IN) (9). Visualization and quantification were as described above.

To demonstrate the effect of the RhoA signaling pathway on ezrin detergent solubility, $10 \mu \mathrm{M}$ of the Rho kinase inhibitorY27632 (11) (Upstate Biotechnology, Lake Placid, NY) was incubated with cells during recovery from depletion (as above) and the ezrin detergent solubility determined as described above. 


\section{Results}

Nucleotide changes during ATP depletion and recovery. Glucose depleted medium plus $100 \mathrm{nM}$ antimycin A was used to induce chemical anoxia in LLC-PK 10 porcine proximal tubule epithelial cells, as a model of ischemic ATP depletion. The relative amount of ATP and GTP were determined by HPLC over the time course for which Rho GTPase activity was also measured (10). The reduction of ATP was rapid (Fig. 1A). Within 5 minutes ATP was almost reduced in half to $52 \%, \pm 17 \%$ of the level in control cells. After 10 minutes of depletion, ATP was reduced to $28 \%, \pm 10.3 \%$ and reached a minimum of $1 \%, \pm 0.4 \%$ of the control value after 60 minutes. The change in GTP followed similar kinetics (Fig. 1B). Within 5 minutes GTP was reduced to less than half, i.e., $62.6 \%, \pm 20.3 \%$ of the control value. After 10 minutes of depletion, GTP was $42.4 \%, \pm 10.8 \%$ and reached a minimum of only $5.8 \%, \pm 3.4 \%$ of the control value after 60 minutes. In addition to a higher relative minimum amount of GTP during ATP depletion conditions, GTP levels recovered more quickly than ATP when the cells were returned to normal growth conditions. GTP levels reached $86.2 \%, \pm 9.4 \%$ of the control value after 60 minutes of recovery in normal medium, while ATP reached only $42.1 \%$, $\pm 9.1 \%$ of the control level after 60 minutes of recovery.

Stress fiber integrity during depletion and recovery. The actin cytoskeleton is remodeled during ATP depletion $(16,17,30)$. Actin stress fibers are regulated by RhoA and found near the basal membrane in $\mathrm{LLCPK}_{10}$ cells (33). During the time course over which we measured ATP and GTP levels, we followed the integrity of stress fibers by labeling F-actin with rhodamine phalloidin (Fig. 2). Normal stress fibers (Fig. 2, 0 minutes) began to break down within 5 minutes after starting antimycin A treatment and 
became progressively more punctate (Fig. 2, 5-90 minutes). After repletion of ATP with incubation in normal medium, stress fibers progressively reorganized (Fig. 2, 90/30 and 90/60 minutes of depletion / repletion).

Measurement of RhoA Activity. A fusion protein (GST-ROK-BD) consisting of glutathione-S-transferase (GST) and the effector-binding domain of human Rho kinase was used to selectively capture the GTP-bound form of RhoA with glutathione-agarose resin (24). Since only the GTP bound form of RhoA binds to the GST-ROK-BD, the active and the inactive proteins could be separated and detected by immunoblotting using anti-RhoA antibody (Fig. 3). We compared the active and inactive RhoA fractions in a control cell detergent lysate (Fig. 3A, lanes 1 and 2) and following 90 minutes of nucleotide triphosphate depletion (Fig. 3A, lanes 7 and 8). After 90 minutes of depletion, the amount of inactive RhoA increased and the amount of active RhoA decreased. The level of GTP-bound RhoA decreased substantially when the cell lysate was incubated with a large excess of exogenous GDP (Fig. 3A, lanes 3 and 4). Glutathione-agarose was incubated with GST as an additional control. Lanes 5 and 6 in figure 3A show that RhoA did not bind to GST-glutathione-agarose non-specifically. Additionally, after 90 minutes of nucleotide depletion, excess exogenous GTP $\gamma$ S (Fig. 3A, lanes 9 and 10) but not GDP (Fig. 3A, lanes 11 and 12) shifted the balance back towards the active form of RhoA. Since removal of the growth factors present in the normal growth medium could affect the activity of RhoA independently of ATP depletion, we tested the effect of removing serum on RhoA activity in control cells not subjected to ATP depletion, and the effect of the presence or absence of serum during the repletion phase. As shown in figure $3 \mathrm{~B}$, the presence or absence of serum during the assay did not affect RhoA activity 
significantly, although in the absence of serum RhoA activity levels during recovery were slightly lower than those observed with serum present. Serum starvation for 24 hours (Fig. 3C) did not significantly alter RhoA activity in these cells, nor did it alter the affect of nucleotide triphosphate depletion.

Following the same time course of stress fiber disruption seen in figure 2, RhoA activity during nucleotide triphosphate depletion and repletion with normal medium is illustrated in figure 4A. Control cells (Fig. 4A, lanes 1 and 2) showed that a large fraction of RhoA was active (bound) in a confluent LLC-PK 10 cell culture. During the time course of nucleotide triphosphate depletion (Fig. 4A, lanes 3-12) the amount of active RhoA decreased. Upon repletion of ATP levels with normal medium the level of active RhoA increased (Fig. 4A, lanes 13-16). The average of three experiments expressed as a ratio of bound protein (active) to unbound protein (inactive) is shown in Fig. 4B. A significant loss of RhoA activity was detected after as little as 5 minutes of nucleotide triphosphate depletion, by which time the activity ratio dropped to $46.2, \pm 15.1 \%$. After 10 minutes of nucleotide triphosphate depletion the activity ratio dropped to $11.4 \%, \pm 8.2$ of the control value. No measurable RhoA activity was detected at 60 minutes of nucleotide triphosphate depletion. Upon repletion with normal medium, the ratio recovered to $83.9 \%, \pm 78.9$ of the control value by 30 minutes.

Measurement of ezrin detergent solubility. To determine whether alterations in the amount of activated RhoA during ATP depletion and recovery were reflected in the behavior of a known cytoskeletal target of Rho signaling, we followed the detergent solubility of the ERM (ezrin, radixin, moesin) protein ezrin as an indication of its association with the actin cytoskeleton during the time course of nucleotide depletion and 
repletion (Fig. 5A). The control detergent fractionation of ezrin ( 0 minutes) shows that under our conditions much of the protein was recovered in the detergent soluble fraction but a significant fraction remained in the detergent insoluble fraction (Fig. 5A, lanes 1 and 2). With nucleotide triphosphate depletion (Fig. 5A, lanes 3-12) the amount of ezrin in the detergent insoluble fraction decreased, suggesting that ezrin had dissociated from the cytoskeleton. As the cells recovered from depletion (Fig 5A, lanes 13-16), the amount of detergent insoluble ezrin increased. An average detergent solubility for ezrin during the time course $(n=3)$ was expressed as the ratio of detergent insoluble to soluble ezrin. The detergent insolubility of ezrin decreased $60 \%$ during nucleotide triphosphate depletion and increased to control level after 30 minutes of repletion with normal medium (Fig. 5B), and showed an overall time course similar to that of RhoA activity with depletion and repletion (Fig. 4B).

The serine/threonine kinase Rho kinase (or ROCK) is activated by binding to RhoA-GTP and phosphorylates ezrin among other targets (28). In order to demonstrate the effect of RhoA signaling on ezrin detergent solubility, we incubated the Rho kinase inhibitor Y27632 (11) with LLC-PK 10 cells during nucleotide triphosphate depletion and repletion. Figure 5C shows a western blot of a typical experiment. In control cells a substantial amount of ezrin was associated with the cytoskeleton in the detergent insoluble fraction (lanes 1 and 2). Following 90 minutes of depletion, ezrin dissociated from the cytoskeleton and a decreased amount was found in the detergent insoluble fraction (lanes 3 and 4). However, with 60 minutes of repletion in normal medium, (lanes 5 and 6) ezrin was again associated with the cytoskeleton in the insoluble fraction. When Y27632 was included in the incubation medium, ezrin failed to re-associate with the 
cytoskeleton after 60 minutes of repletion, suggesting that Rho kinase, and therefore RhoA signaling, is important for the stable association of ezrin with the cytoskeleton. Activity measurement of Cdc42 and Racl. The depletion of nucleotide triphosphates likely affects the activity of the other Rho family GTPases as well as RhoA itself. We measured the activity of Cdc42 and Rac1 with a similar pull-down assay using a fusion of GST to the effector domain of Pak (containing the CRIB domain) to selectively bind GTP-bound Rac1 and Cdc42. To validate the assay, nucleotides were added to detergent extracts of LLC-PK 10 cells to change the activity of the GTPases (Fig. 6A). Without exogenously added nucleotides (Fig. 6A, lanes 1-2) most of Rac1 and nearly all of Cdc42 was found in the bound (active) lane. The addition of GDP increased the amount of GTPase in the unbound (inactive) fraction for both Cdc42 and Rac1. GST alone (Fig. 6A, lanes 5 and 6) did not pull-down any GTPase. When the cells were first depleted for 90 minutes with antimycin A, Cdc42 did not show any significant decrease in its activity. The level of active Rac1 was decreased after 90 minutes of depletion, but a significant fraction of GTP-bound Rac1 remained (Fig. 6A, lanes 7 and 8). Incubation with GTP $\gamma \mathrm{S}$ increased the amount of GTPase in the active fraction for both proteins (Fig. 6A, lanes 9 and 10). Addition of GDP decreased the total amount of Cdc42 that was recovered, making it impossible to determine the effect of GDP on the activity of Cdc42 in this in vitro assay. GDP decreased the amount of active Rac1 (Fig. 6A, lanes 11 and 12). We tested whether serum deprivation contributed to the effects we observed. Substituting serum free medium for normal growth medium, without induction of ATP depletion (Fig. 6B, lanes 1-4) did not affect Rac1 or Cdc42 activity, nor did it alter the effect of ATP depletion (Fig. 6B, lanes 5 and 6). Serum starvation (24 hours) had no 
appreciable effect on Rac1 activity, but did result in a decrease in Cdc42 activity (Fig. 6C lanes 1 and 2). However, Cdc42 and Rac1 maintained high levels of activity after 90 minutes of ATP depletion, even with prior serum starvation (Fig 6C, lanes 9 and 10).

Since, in contrast to our results with RhoA (Fig. 3, lanes 7 and 8 and Fig. 4), there was no significant decrease in the amount of active Cdc42 and Rac1 with depletion we sought to demonstrate that the GTPase assay was capable of detecting physiological changes in the activity of Rac1 and Cdc42 in LLC-PK cells. Since cadherin mediated cell-cell adhesion has been shown to result in alterations in Rho GTPase activity (32), the activities of RhoA, Rac1 and Cdc42 were assayed using the pull-down assay 24 hours after seeding LLC-PK 10 cells at a range of different densities. A representative western blot is shown in figure 7A. RhoA activity increased more than 36-fold from the lowest to the highest initial cell density (graphed in figure 7B), while Rac1 and Cdc42 activity decrease by $91.1 \%, \pm 6.5 \%$ and $85.2 \%, \pm 14.7 \%$ respectively. These experiments showed that our assay conditions were sufficiently sensitive to have detected physiological changes in the activity of all three GTPases during ATP depletion and recovery.

A comparison of the changes in the activity of RhoA, Rac1 and Cdc42 during 90 minutes of nucleotide triphosphate depletion and depletion, followed by 60 minutes of repletion in normal medium is shown in figure $8 \mathrm{~A}$. Under normal culture conditions $(\mathrm{t}=0)$ in post-confluent cultures all three GTPases were active (B, bound fraction). While RhoA was completely inactive after 90 minutes of depletion (U, unbound fraction), Rac1 was still active and Cdc42 activity had changed negligibly. A more complete time course of nucleotide triphosphate depletion and repletion for Rac1 activity revealed that Rac1 activity dropped after initiation of depletion to a minimum at 30 minutes, but rebounded 
to higher levels after 60 and 90 minutes (Fig. 8B). With repletion Rac1 activity increased to control levels after 30 minutes.

The increase in Rac1 activity after an initial decrease during depletion raised the possibility that Rac1, RhoA or Cdc 42 activity may fluctuate over a longer time course. However, a time course extended to 240 minutes of nucleotide triphosphate depletion showed that the activity of each of the three GTPases was relatively constant over longer time periods. RhoA activity remained near zero, Rac1 activity remained steady during 90 to 240 minutes of depletion and Cdc42 remained almost entirely in the active state (Fig. 9).

Effect of activated RhoA during depletion. Considerable evidence exists for cross talk between the activities of different Rho family GTPases (22). Since RhoA activity decreases so rapidly with nucleotide depletion we considered that this might affect the activity of Rac1 or Cdc42. To test this possibility, the constitutively active RhoA mutant RhoAL63 was transfected into LLC-PK 10 cells. Subsequently, nucleotide triphosphate depletion was induced with antimycin A and the activities of RhoA, Rac1 and Cdc42 were measured after 90 minutes of depletion and after depletion followed by 60 minutes of repletion. No effect was found on the activity of endogenous RhoA or Cdc42 (data not shown). However, the ratio of active to inactive Rac1 decreased $32.1 \%$ during repletion, in the presence of RhoAL63 compared to untransfected controls. However, this change did not reach statistical significance $(n=3, P=0.189)$. 


\section{Discussion}

We demonstrate in this report that chemically induced anoxia in a cultured cell line derived from renal proximal tubule results in rapid conversion of RhoA-GTP to RhoA-GDP, with a time course that parallels the decrease in ATP and GTP levels. Repletion of ATP and GTP resulted in corresponding reversal of this effect and increasing levels of RhoA-GTP. The kinetics of RhoA inactivation and re-activation during ATP depletion and repletion were consistent with the cytoskeletal association of the known RhoA effector ezrin through depletion and repletion, which we show here and which is consistent with previous reports $(8,9)$. We used the Rho kinase inhibitor Y27632 to show the importance of RhoA signaling and the specific effect of Rho kinase inhibition on ezrin cytoskeletal association. In these experiments, Y27632 prevented the reassociation of ezrin with the cytoskeleton during ATP repletion. Myosin II regulatory light chain phosphorylation, another downstream target of RhoA signaling, also changes during ATP depletion with kinetics consistent with the changes we observe in RhoA activity (34). This pattern of RhoA inactivation in ischemia is consistent with the finding that constitutively active RhoA mutants are able to protect components of the actin cytoskeleton and associated structures in the basal and lateral domains of the cell cortex against disruption during ATP depletion $(17,33)$, and confirms an important role for RhoA in the pathophysiology of ischemic injury in the kidney.

In contrast to the effect of ATP depletion on RhoA, the activation state of Rac1 and Cdc42 did not directly correlate with the level of ATP or GTP in the cell. We confirmed that our assay was sensitive to physiological changes in Rac1 and Cdc42 activity by measuring large decreases in activity as cell density increased to confluence. It 
is interesting to note that the changes we observed in RhoA, Rac1 and Cdc42 activity were in the opposite direction to those observed in similar experiments on cell density in another renal epithelial cell line, MDCK (32). This discrepancy is not necessarily surprising, since other work has shown that signaling pathways involving Rho GTPases differ between MDCK and LLC-PK cells (S. Gopalakrishnan, MAH, SJA and J.A. Marrs, in press). It should be noted that the assays we use here are valid as an indication of relative changes in GTPase activity that result from the manipulations we used to alter ATP levels or cadherin engagement, but cannot be used as an indication of whether the basal level of GTPase activity in these cells is high or low compared to studies in other cells by other investigators, due to the sensitivity of the assay to buffer conditions and other variables in the protocol used.

Our assay showed that Cdc42 activity did not decrease during ATP depletion, while the level of active Rac1 initially declined, but later recovered somewhat, even as ATP and GTP levels were still falling. Disruption of actin cytoskeletal organization is not, therefore, attributable to a net decrease in the cellular level of Cdc42 activity, and cannot be related in a simple way to the level of Rac1 activity. It may be that the activity of Cdc42 and Rac1 is still an important factor in cytoskeletal disruption, but that this disruption results instead from an imbalance in the level of activity of these Rho GTPases. In the absence of detectable RhoA activity, continued Cdc42 and Rac1 activity might indeed be one of the causes of aberrant actin polymerization into F-actin aggregates in the center of the cell that characterizes ATP depletion in renal proximal tubule cells (31), as well as in other cell types, such as fibroblasts and endothelial cells $(14,20)$. Actin monomers released by the destruction of normal cytoskeletal assemblies 
following RhoA inactivation, and from the thymosin sequestered pool, could be induced to polymerize at nuclei provided by the activity of Rac1 and $\mathrm{Cdc} 42$ effectors such as cortactin $(38,41)$.

The partial recovery of Rac1 activity at later times during ATP depletion, when ATP and GTP levels are still falling, is surprising. Since in many cell types RhoA activity antagonizes that of $\operatorname{Rac} 1(23,39)$, we considered that the nearly complete inactivation of RhoA could explain the partial recovery of Rac1. However, the constitutively active RhoA mutant RhoAL63 had no effect in Rac1 activity under ATP depleted conditions, so the signaling relays that normally operate between the two GTPases appear to be disrupted by ATP depletion, but are again operable during repletion.

Under the conditions used here for ATP depletion, cellular ATP levels decrease to about $1 \%$ of the level in control cells, while the levels of GTP decrease to a minimum of $5.8 \%$ of control levels. Assuming that the normal concentrations of ATP and GTP in our LLC-PK 10 cells are comparable to other cell types (26), this corresponds to a decrease in ATP concentration from 2-5 $\mathrm{mM}$ to $20-50 \mu \mathrm{M}$, and a decrease in the GTP concentration from $\sim 300 \mu \mathrm{M}$ to $\sim 17 \mu \mathrm{M}$. These GTP concentrations still significantly exceed the affinity constants of the GTPases for GTP. RhoA, Rac1 and Cdc42 GTPases' affinities for GTP are comparable $\left(\mathrm{K}_{\mathrm{D}} \sim 0.2 \mu \mathrm{M}\right)$, as are their slightly lower affinities for GDP $\left(\mathrm{K}_{\mathrm{D}} \sim 0.5-0.6 \mu \mathrm{M}\right)(43)$. Exchange factors (GEFs) act by increasing the dissociation rate constant for bound nucleotide, so that in normal cells the cytoplasmic excess of GTP over GDP ensures that when GEFs bind the result is formation of the GTP-bound form of the protein. Under depleted conditions, however, GDP is in excess over GTP (at least over the short time scales as used here), and the activity of exchange factors could be expected 
to result in inactivation of Rho proteins, since the relative affinities for GTP and GDP do not bias the exchange reaction sufficiently to prevent this. This may well be what happens to RhoA since the rapid kinetics of RhoA inactivation that we observe would be hard to account for by the intrinsic dissociation or hydrolysis rates, and must therefore result from the action of GEFs or GAPs. A role for regulatory proteins also seems to be required in view of the very different behavior of RhoA, Rac1 and Cdc42. If alterations in Rho protein activity were simply the result of their equilibration to the ambient GTP:GDP ratio in the cytoplasm one would expect to see the same effect of ATP depletion on all three GTPases, since their nucleotide affinities, dissociation rates and hydrolysis rates are comparable. That the effect of ATP depletion is so different for each protein argues that depletion selectively impacts the regulation of upstream pathways that control the activities of the GEF and GAP proteins. These upstream pathways may still directly sense the GTP:GDP or ATP:ADP ratio, but could transduce the effects of alterations in these ratios differentially to each Rho family member. Since it is generally accepted that GAP proteins are more promiscuous and GEF proteins more specific, it is reasonable to suppose that the differences in the effects of ATP depletion on RhoA, Rac1 and $\mathrm{Cdc} 42$ result from different effects on specific GEF proteins. 


\section{Acknowledgements}

This work was supported by the National Institute of Diabetes, Digestive and Kidney Diseases Grant DK-53194 (S.J. Atkinson). Facilities used for confocal imaging in the Indiana Center for Biological Microscopy are supported by a grant from the Lilly Endowment (INGEN) to the Indiana University School of Medicine, and are a core resource for the George O'Brien Center for Advanced Renal Microscopy (NIDDK Grant DK-61594).

We thank Jing Chen, Gary Bokoch and Kozo Kaibuchi for reagents; Cathy Cox, Zoya Plotkin and Matt Loesch for technical assistance; and Bruce Molitoris, Tim Sutton and Jim Marrs for valuable discussion. 


\section{References}

1. Algrain M, Turunen, O, Vaheri A, Louvard D, and Arpin M. Ezrin contains cytoskeleton and membrane binding domains accounting for its proposed role as a membrane-cytoskeletal linker. J. Cell Biol. 120: 129-139, 1993.

2. Au JL, Su MH, and Weintjes MG. Extraction of intracellular nucleosides and nucleotides with acetonitrile. Clin. Chem. 35: 48-51, 1989.

3. Bacallao R, Garfinkel A, Monke S, Zampighi G, and Mandel LJ. ATP depletion: a novel method to study junctional properties in epithelial tissues. I. Rearrangement of the actin cytoskeleton. J. Cell Sci. 107: 3301-3313, 1994.

4. Benard V, Bohl BP, and Bokock GM. Characterization of Rac and Cdc42 activation in chemoattractant-stimulated human neutrophils using a novel assay for active GTPases. J. Biol. Chem. 274: 13198-13204, 1999.

5. Benard V, and Bokoch GM. Assay of Cdc42, Rac, and Rho GTPases activation by affinity methods. Methods Enzymol. 345: 349-359, 2002.

6. Bishop AL, and Hall A. Rho GTPases and their effector proteins. Biochem. J. 348: $241-255,2000$.

7. Bokoch GM. Regulation of cell function by Rho family GTPases. Immunologic Res. 21: 139-148, 2000.

8. Chen J, Cohn JA and Mandel LJ. Dephosphorylation of ezrin as an early event in renal microvillar breakdown and anoxic injury. Proc. Natl. Acad. Sci., USA. 92: 74957499. 1995. 
9. Chen J, Doctor BR, and Mandel LJ. Cytoskeletal dissociation of ezrin during renal anoxia: role in microvillar injury. Am. J. Physiol. (Cell Physiol.) 267: C784-C795, 1994. 10. Dagher PC. Modeling ischemia in vitro: selective depletion of adenine and guanine nucleotide pools. Am. J. Physiol. (Cell Physiol.): 279: C1270-C1277, 2000.

11. Davies SP, Reddy H, Caivano M, and Cohen P. Specificity and mechanism of action of some commonly used protein kinase inhibitors. Biochem. J. 351: 95-105, 2000.

12. De Vendittis E, Zahn R, and Fasano O. Regeneration of the GTP-bound from the GDP-bound form of human and yeast ras protein by nucleotide exchange. Stimulatory effect of organic and inorganic polyphosphates. Eur. J. Biochem. 161: 473-478, 1986. 13. Edelstein CL, Ling H, and Schrier RW. The nature of renal cell injury. Kidney Int. 51: 1341-1351, 1997.

\section{Glascott PA Jr, McSorley KM, Mittal B, Sanger JM, and Sanger JW. Stress} fiber reformation after ATP depletion. Cell Motil. Cytoskeleton 8: 118-29, 1987.

15. Glaumann B, Glaumann H, Brerezesky IK, and Trump BF. Studies on the pathogenesis of ischemic cell injury. II. Morphological changes of the pars convoluta (P1 and P2) of the proximal tubule of the rat kidney made ischemic in vivo. Virchows Arch. 19: 281-302, 1975.

16. Gopalakrishnan S, Dunn KW, and Marrs JA. Rac1, but not RhoA, signaling protects epithelial adherens junction assembly during ATP depletion. Am. J. Physiol. (Cell Physiol.) 283: C261-C272, 2002.

17. Gopalakrishnan S, Raman N, Atkinson SJ, and Marrs JA. Rho GTPase signaling regulates tight junction assembly and protects tight junctions during ATP depletion. Am. J. Physiol. (Cell Physiol. 44) 275: C798-C809, 1998. 
18. Grune T, Seims W, Gerber G, Tikhonov YV, Pimenov AM, and Toguzov RT. Changes in nucleotide patterns in liver, muscle and blood during the growth of Ehrlich ascites cells: application of the reversed-phase and the ion-pair reversed-phase highperformance liquid chromatography with radial compression column. J. Chromatogr. 563: 53-61, 1991.

19. Hanzel D, Reggio H, Bretsher A, Forte JG, and Mangeat P. The secretionstimulated $80 \mathrm{~K}$ phosphoprotein of parietal cells is ezrin, and has properties of a membrane cytoskeletal linker in the induced apical microvilli. EMBO J. 10: 2363-2373, 1991.

20. Hinshaw DB, Burger JM, Miller MT, Adams JA, Beals TF, and Omann GM. ATP depletion induces an increase in the assembly of a labile pool of polymerized actin in endothelial cells. Am. J. Physiol. (Cell Physiol.) 264: C1171-1179, 1993.

\section{Hirao M, Sato N, Kondo T, Yonemura S, Monden M, Sasaki T, Takai Y,} Tsukita Sh, and Tsukita Sa. Regulation mechanism of ERM (ezrin, radixin, moesin) protein/plasma membrane association: Possible involvement of phosphatidylinositol turnover and Rho-dependent signaling pathway. J. Cell Biol. 135: 37-51, 1996.

22. Kjoller L, and Hall A. Signaling to Rho GTPases. Exp. Cell Res. 253: 166-179, 1999.

23. Kozma R, Sarner S, Ahmed S, and Lim L. Rho family GTPases and neuronal growth cone remodeling: relationship between increased complexity induced by Cdc42Hs, Rac1 and acetylcholine and collapse induced by RhoA and lysophosphatidic acid. Mol. Cell Biol. 17: 1201-1211, 1997. 


\section{Kraenburg O, Poland M, van Horck FPG, Drechsel D, Hall A, and Moolenaar}

WH. Activation of RhoA by lysophosphatidic acid and $\mathrm{G \alpha}_{12 / 13}$ subunits in neuronal cells: Induction of neurite retraction. Mol. Biol. Cell 10: 1851-1857, 1999.

25. Madaule P, and Axel R. A novel ras-related gene family. Cell 41: 31-40, 1985.

26. Mathews C, and Van Holde KE. Biochemistry. Melno Park, CA: The Benjamin / Cummings Publishing Company, Inc.1996, p.425.

27. Matsui T, Amano M, Yamamoto T, Chihara K, Nakafuku M, Ito M, Nakano T, Okawa K, Iwamatsu A, and Kaibuchi K. Rho-associated kinase, a novel serine / threonine kinase, as a putative target for small GTP binding protein Rho. EMBO J. 15: 2208-2216, 1996.

28. Matsui T, Maeda M, Doi Y, Yonemura S., Amano M, Kaibuchi K, Tsukita Sa, and Tsukita Sh. Rho kinase phosphorylates $\mathrm{COOH}$-terminal threonines of ezrin/radixin/moesin (ERM) proteins and regulates their head-to-tail association. J. Cell Biol. 140: 647-657, 1998.

29. Matsui T, Yonemura S, Tsukita Sh, and Tsukita Sa. Activation of ERM proteins in vivo by Rho involves phosphatidylinositol- 4-phosphate 5-kinase and not ROCK kinases. Curr. Biol. 9: 1259-1262, 1999.

30. Molitoris BA, Geerdes A, and McIntosh JR. Dissociation and redistribution of the $\mathrm{Na}+, \mathrm{K}(+)$-ATPase from its surface membrane actin cytoskeletal complex during cellular ATP depletion. J. Clin. Invest. 88: 462-469, 1991.

31. Molitoris BA, and Nelson WJ. Alterations in the establishment and maintenance of epithelial cell polarity as a basis for disease processes. J. Clin. Invest. 85: 3-9, 1990. 
32. Noren NK, Niessen CM, Gumbiner BM, and Burridge K. Cadherin engagement regulates Rho family GTPases. J. Biol. Chem. 276:33305-8, 2001.

33. Raman N, and Atkinson SJ. Rho controls actin cytoskeletal assembly in renal epithelial cells during ATP depletion and recovery. Am. J. Physiol. (Cell Physiol. 45) 276: C1312-C1324, 1999.

34. Sutton TA, Mang HE, and Atkinson SJ. Rho-kinase regulates myosin II activation in MDCK cells during recovery after ATP depletion. Am. J. Physiol. (Renal Physiol.) 281: F810-F818, 2001.

35. Takeuchi K, Sato N, Kashiara H, Funayama N, Nagafuchi A, Yonemura S, Tsukita Sa, and Tsukita Sh. Perturbation of cell adhesion and microvilli formation by antisense oligonucleotides to ERM family members. J. Cell Biol. 125: 1371-1384, 1994.

36. Tsukita S, Oishi K, Sato N, Sargara J, Kawai A, and Tsukita S. ERM family members as molecular linkers between the cell surface glycoprotein CD44 and the actinbased cytoskeleton. J. Cell Biol. 126: 391-401, 1994.

37. Turunen O, Wahlstrom T, and Vaheri. A. Ezrin has a $\mathrm{COOH}$-terminal actin binding site that is conserved in the ezrin protein family. J. Cell Biol. 126: 1445-1453, 1994.

38. Uruno T, Liu J. Zhang P, Fan Y, Egile C, Li R, Mueller SC, and Zhan X. Activation of Arp2/3 complex-mediated actin polymerization by cortactin. Nature Cell Biol. 3: 259-266, 2001.

39. Van Leeuwen FN, Kain HET, van der Kammen RA, Michiels F, Kranenburg OW, and Collard JG. The guanine nucleotide exchange factor Tiam1 affects neuronal 
morphology: opposing roles for the small GTPases Rac and Rho. J. Cell Biol. 139: 797807, 1997.

40. Venkatachalam MA, Jones DB, Rennke HG, Sandstrom D, and Patel Y. Mechanisms of proximal tubule brush border loss and regeneration following mild renal ischemia. Lab Invest. 45: 355-365, 1981.

\section{Weaver AM, Karginov AV, Kinley AW, Weed SA, Li Y, Parsons JT, and}

Cooper JA. Cortactin promotes and stabilizes Arp2/3-induced actin filament network formation. Curr. Biol. 11: 370-374, 2001.

42. White P, Doctor RB, Dahl RH, and Chen J. Coincident microvillar actin bundle disruption and perinuclear sequestration in anoxic proximal tubule. Am. J. Physiol. (Renal Physiol.) 278: F886-F893, 2000.

43. Zhang $\mathbf{B}$, Zhang $\mathbf{Y}$, Wang $\mathbf{Z}$, and Zheng $\mathbf{Y}$. The role of $\mathrm{Mg}^{2+}$ cofactor in the guanine nucleotide exchange and GTP hydrolysis reactions of Rho family GTP-binding proteins. J. Biol. Chem. 275: 25299-25307, 2000.

Rho Activity and Ischemia 


\section{Figure legends}

Fig. 1. Nucleotide triphosphate levels during depletion and recovery measured by HPLC. Values are means $\pm S D, n=3$. Cells were treated with antimycin $A(0.1 \mu M)$ and substrate depleted media for the indicated times $(5,10,30,60$ and 90 minutes) to induce depletion, or depleted for 90 minutes and returned to normal media for 30 or 60 minutes $(90 / 30$, 90/60) A: Change in ATP level as a percentage of the control (0 minutes). B: Corresponding values for GTP.

Fig. 2. Effect of nucleotide triphosphate depletion on stress fiber morphology. Confocal fluorescence micrographs show LLC-PK 10 cells stained with rhodamine phalloidin to label filamentous actin. Cells were treated with antimycin A and substrate depleted medium for $0,5,10,30,60$ and 90 minutes, or treated for 90 minutes and returned to normal medium. Magnification is $100 \mathrm{X}$.

Fig. 3. Western blot showing assay for RhoA activity. A. LLC-PK 10 cells were incubated for 0 or 90 minutes with antimycin A and substrate depleted medium. The indicated nucleotides were added to some lysates, and all lysates were then incubated with GST-ROK-BD or GST alone (indicated) and separated into bound fraction (B), containing RhoA-GTP or unbound fraction (U), containing RhoA-GDP by incubation and centrifugation with glutathione agarose resin. Each fraction was analyzed by western blotting with an anti-RhoA antibody. B. Comparison of RhoA activity with serum present in the control (time 0 , lanes 1 and 2) or with serum removed for 90 minutes (lanes 3 and 4), and effect of ATP repletion in the presence (lanes 7 and 8) and absence (lanes 9 and 
10) of serum. C. Cells were serum starved for 24 hours prior to ATP depletion (serum starvation) or fed with fresh serum containing medium 24 hours prior to the experiment (no starvation).

Fig. 4. Effect of depletion and recovery on RhoA activity. A. LLC-PK 10 cells were incubated with antimycin A for various lengths of time or incubated with antimycin A for 90 minutes followed by recovery in normal medium for 30 or 60 minutes. Detergent lysates were then incubated with GST-ROK-BD and separated into bound (B), containing RhoA-GTP or unbound (U), containing RhoA-GDP fractions by incubation and centrifugation with glutathione agarose. B. Graph of three experiments as represented in A. The amount of RhoA in each fraction was quantified by densitometry of western blots and expressed as the mean ratio, \pm S.D., of bound to unbound as a percentage of the control $(t=0)$ value.

Fig. 5. Effect of depletion and recovery on cytoskeletal association of ezrin. A. LLC$\mathrm{PK}_{10}$ cells were incubated with antimycin A for various lengths of time or incubated with antimycin A for 90 minutes followed by recovery in normal medium for 30 or 60 minutes. After detergent lysates (S) were made for the RhoA activity assay (Fig. 4), the detergent insoluble fractions were solubilized with SDS (I) and analyzed by western blot using an anti-ezrin polyclonal antibody. B. Graph of three experiments as represented in A. The amount of ezrin in each fraction was quantified by densitometry of the western blots and expressed the mean ratio, \pm S.D., of detergent insoluble to detergent soluble ezrin. C. Western blot showing a typical experiment of the effect of the Rho kinase 
inhibitor Y27632 on ezrin detergent solubility during recovery from ATP depletion.

LLC-PK $_{10}$ cells were incubated with antimycin A for 0 or 90 minutes or 90 minutes with 60 minutes of repletion with or without Y27632. (S) is detergent soluble and (I) detergent insoluble ezrin.

Fig. 6. Western blot showing assay for Cdc42 and Rac1 activity. LLC-PK 10 cells were either incubated for 0 or 90 minutes with antimycin A. Detergent lysates were incubated with the indicated nucleotides. All lysates were then incubated with GST-Pak-BD or GST alone (indicated) and separated into bound (B), containing GTP-bound or unbound (U), containing GDP-bound GTPase fractions by incubation and centrifugation with glutathione agarose. Rac1 and $\mathrm{Cdc} 42$ in the bound and unbound fractions were detected using specific antibodies. B. Comparison of Rac1 and Cdc42 activity with serum present in the control (time 0, lanes 1 and 2) or with serum removed for 90 minutes (lanes 3 and 4), and effect of ATP repletion in the presence (lanes 7 and 8) and absence (lanes 9 and 10) of serum. C. Cells were serum starved for 24 hours prior to ATP depletion (serum starvation) or fed with fresh serum containing medium 24 hours prior to the experiment (no starvation).

Fig. 7. Effect of increasing cell density on the activities of RhoA, Rac1 and Cdc42. A. LLC-PK 10 cells were plated at cell densities increasing in a geometric progression by a factor of two. After 24 hours, activity assays $(n=3)$ were performed for each GTPase. Bound (B), contains GTP-bound and unbound (U) contains GDP-bound GTPase. B. Western blots were quantified by densitometry. The activities are expressed as the 
percent change in the mean ratio, \pm S.D., of bound to unbound GTPase between the ratios measured at the lowest and highest cell densities.

Fig. 8. A. Comparison of RhoA, Rac1 and Cdc42 activity assays. LLC-PK 10 cells were treated with antimycin A and substrate depleted medium for 90 minutes or 90 minutes of antimycin A, followed by recovery in normal medium for 60 minutes. B. Effect of depletion and recovery on Rac1 activity. LLC-PK 10 cells were incubated with antimycin A and substrate depleted medium for various lengths of time indicated or incubated with antimycin A for 90 minutes followed by recovery in normal medium for 30 or 60 minutes. Detergent lysates were then incubated with GST-Pak-BD and separated into bound (B), containing GTP-Rac1 or unbound (U), containing GDP-Rac1 fractions by incubation and centrifugation with glutathione agarose. C. Graph of four experiments as represented in B. The amount of Rac1 in each fraction was quantified by densitometry of western blots and expressed as the mean ratio, \pm S.D., of bound to unbound as a percentage of the control $(\mathrm{t}=0)$ value.

Fig. 9. Extended time course of GTPase activity. LLC-PK 10 cells were treated with antimycin A for 0, 90, 180 or 240 minutes. The cells were lysed and GTPase activities were measured as described previously. 
Figure 1

A

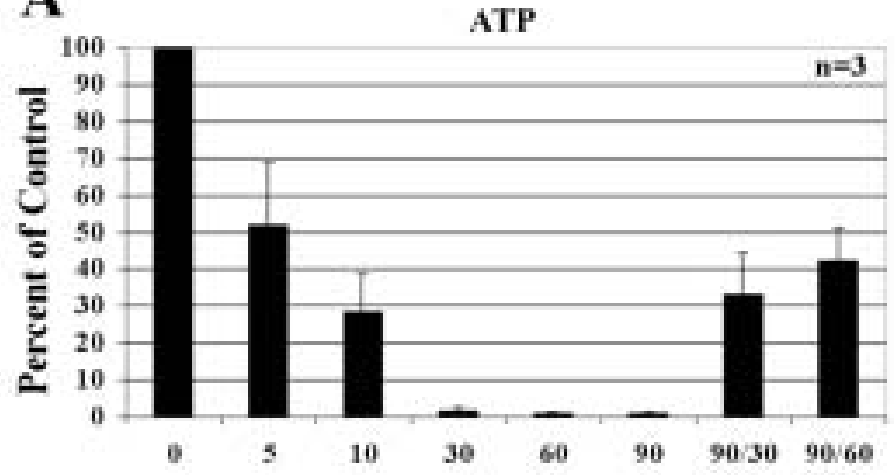

Time of Depletion / Repletion (minutes)

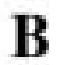

GTP

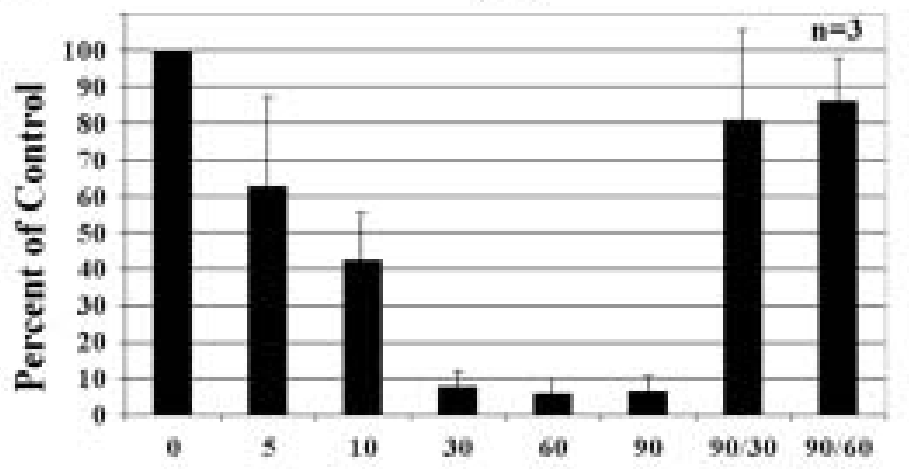

Time of Depletion / Repletion (minutes)

Rho Activity and Ischemia 
Figure 2

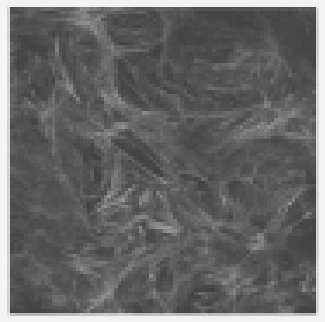

0

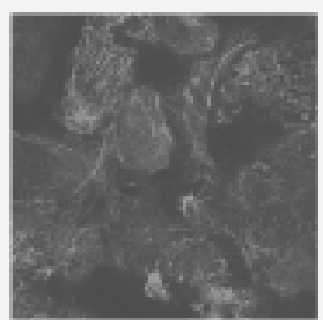

60

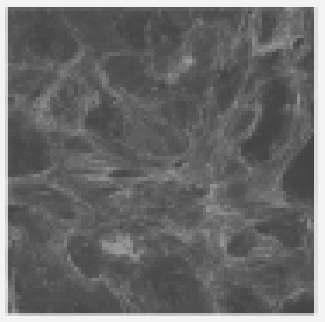

5

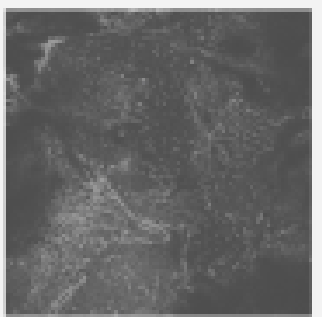

90

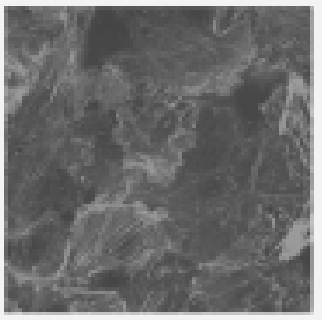

10

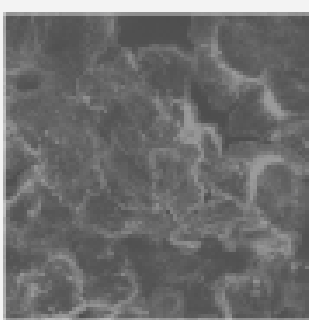

$90 / 30$

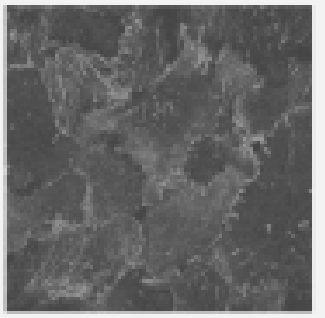

30

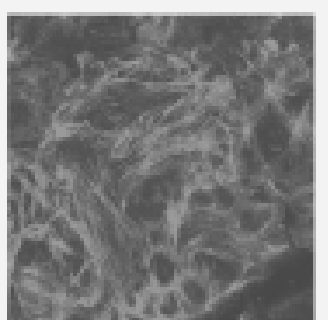

$90 / 60$ 


\section{Figure 3}

A

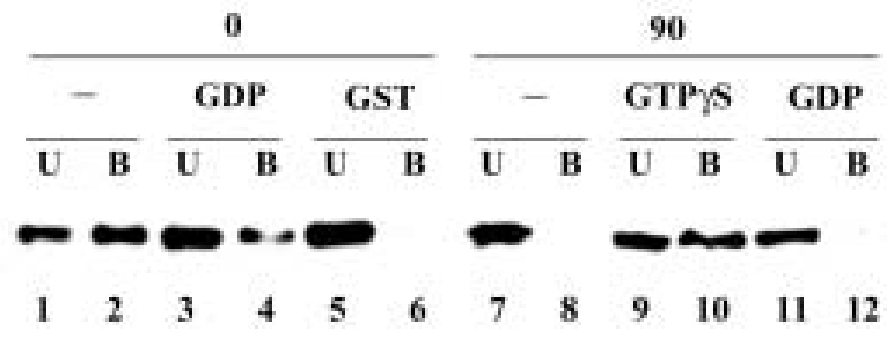

B

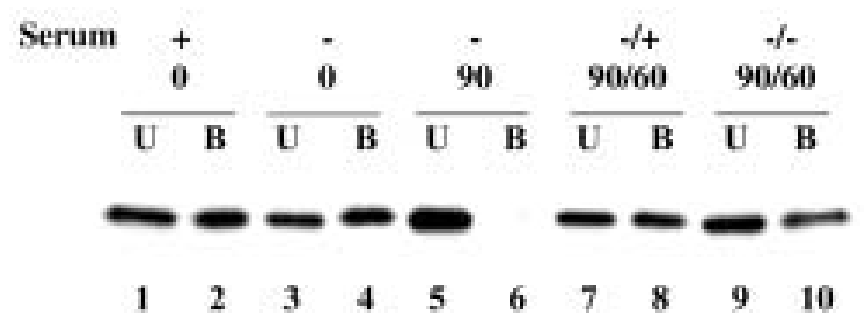

C

\begin{tabular}{|c|c|c|c|c|c|c|c|c|c|}
\hline \multicolumn{6}{|c|}{ No Starvation } & \multicolumn{4}{|c|}{ Serum Starvation } \\
\hline & & & & & & & 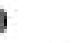 & 90 & $90 / 60$ \\
\hline U & B & U & B & U & B & U & B & U B & $\mathbf{U}$ \\
\hline - & - & - & & - & - & $\sim$ & 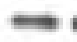 & 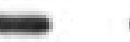 & $-\infty$ \\
\hline 1 & 2 & 3 & 4 & 5 & 6 & 7 & 8 & 10 & $11 \quad 12$ \\
\hline
\end{tabular}

Rho Activity and Ischemia 


\section{Figure 4}

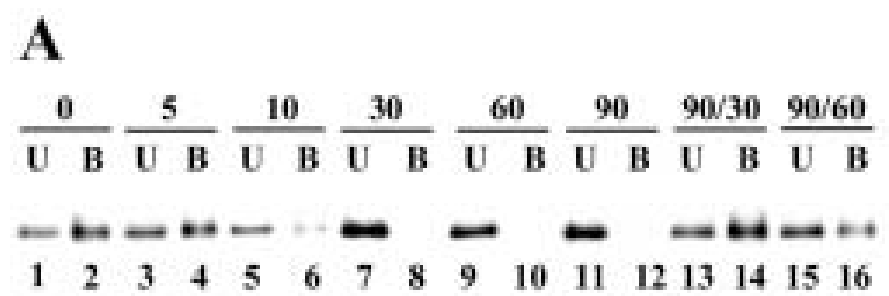

B

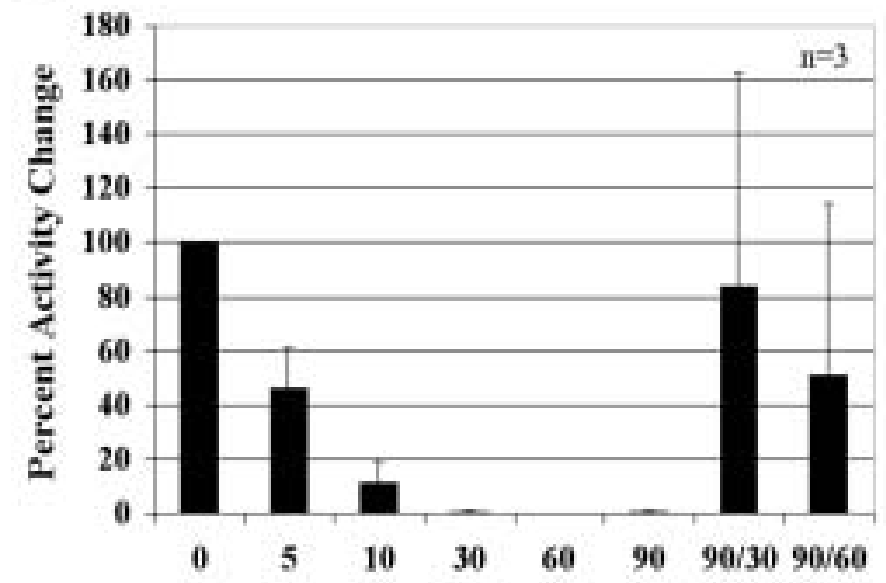

Time of Depletion / Repletion (minutes)

Rho Activity and Ischemia 
Figure 5

A

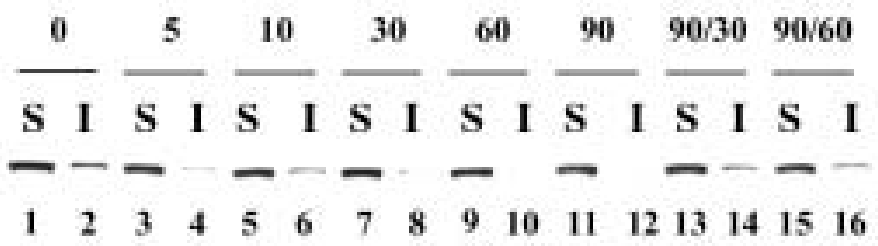

B

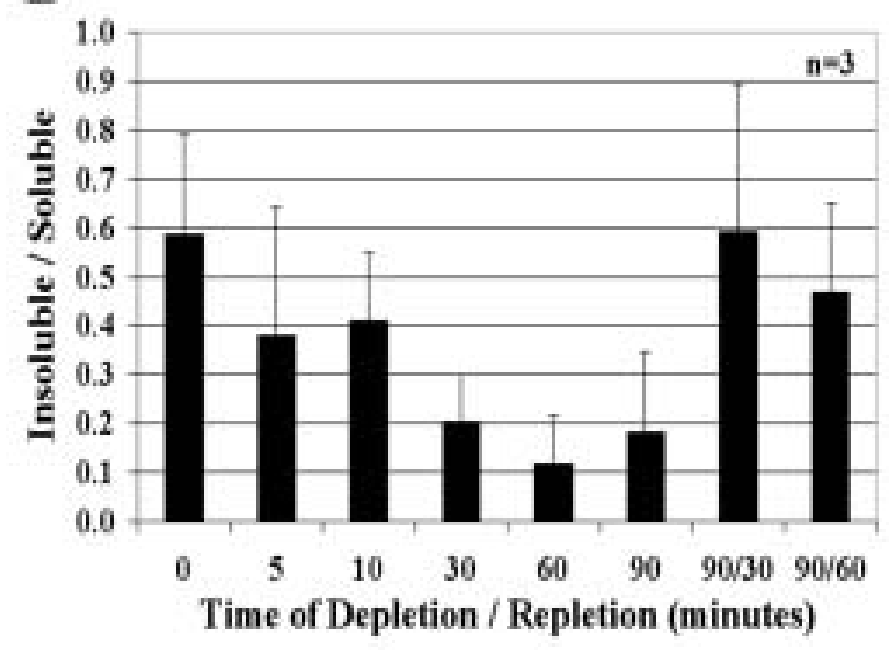

C

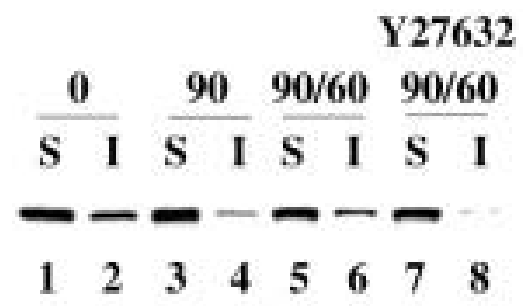

Rho Activity and Ischemia 
Figure 6

A

\begin{tabular}{|c|c|c|c|c|}
\hline \multicolumn{3}{|c|}{0} & \multicolumn{2}{|l|}{90} \\
\hline - & GDP & GST & GTPYS & GDP \\
\hline
\end{tabular}

\section{Cde42}

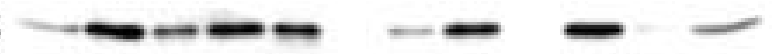

Rac1

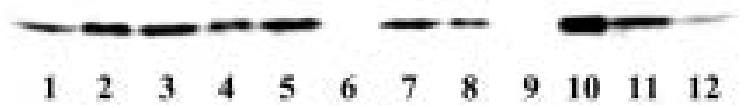

B

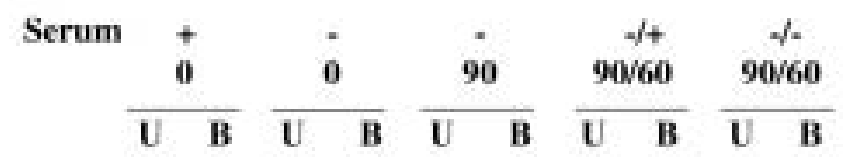

Cde42

Rac1

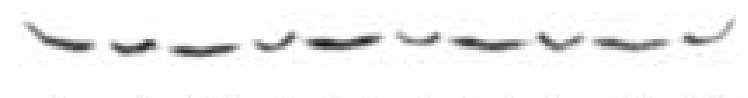

$\begin{array}{llllllllll}1 & 2 & 3 & 4 & 5 & 6 & 7 & 8 & 9 & 10\end{array}$

C

\begin{tabular}{|c|c|c|c|c|c|}
\hline \multicolumn{3}{|c|}{ No Starvation } & \multicolumn{3}{|c|}{ Serum Starvation } \\
\hline 0 & 90 & $90 / 60)$ & 0 & 90 & $90 / 60$ \\
\hline
\end{tabular}
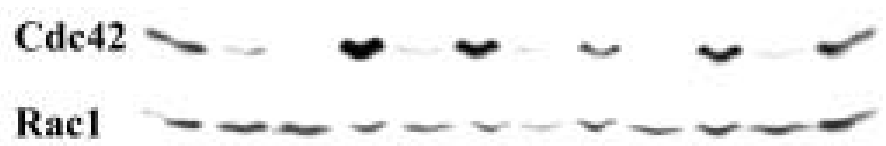

$\begin{array}{llllllllllll}1 & 2 & 3 & 4 & 5 & 6 & 7 & 8 & 9 & 10 & 11 & 12\end{array}$

Rho Activity and Ischemia 
Figure 7

\begin{tabular}{|c|c|c|c|c|c|c|c|}
\hline \multicolumn{5}{|c|}{ Increasing cell density } & \multicolumn{3}{|c|}{ Confluent } \\
\hline U & B & v & B & $\mathbf{U}$ & B & U & B \\
\hline
\end{tabular}

RhoA

Rac1

Cde42

B
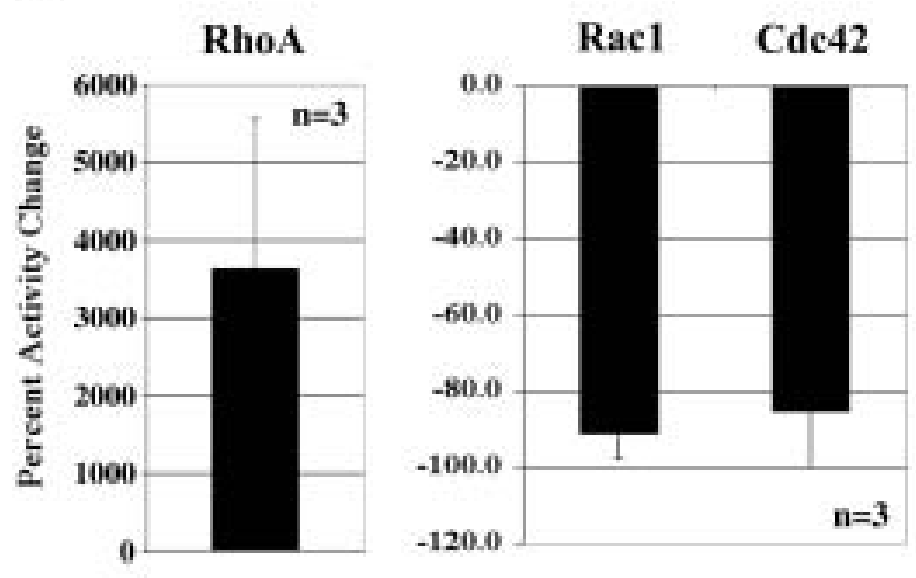

Rho Activity and Ischemia 
Figure 8

A

$\frac{0}{U^{\prime} \text { B }} \frac{90}{\text { U B B U B }}$

RhoA

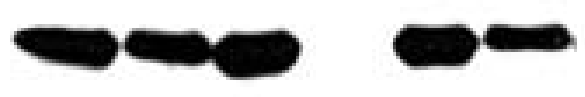

Rac1

Cde42

B

$\frac{0}{U \text { B }} \frac{5}{U \text { B }} \frac{10}{\text { B }} \frac{30}{U \text { B }} \frac{60}{U \text { B }} \frac{90}{\text { B }} \frac{90 / 30}{U \text { B }} \frac{90 / 60}{\text { B }}$

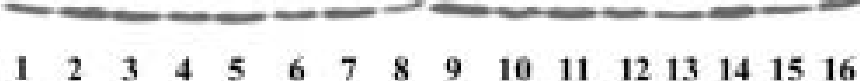

C

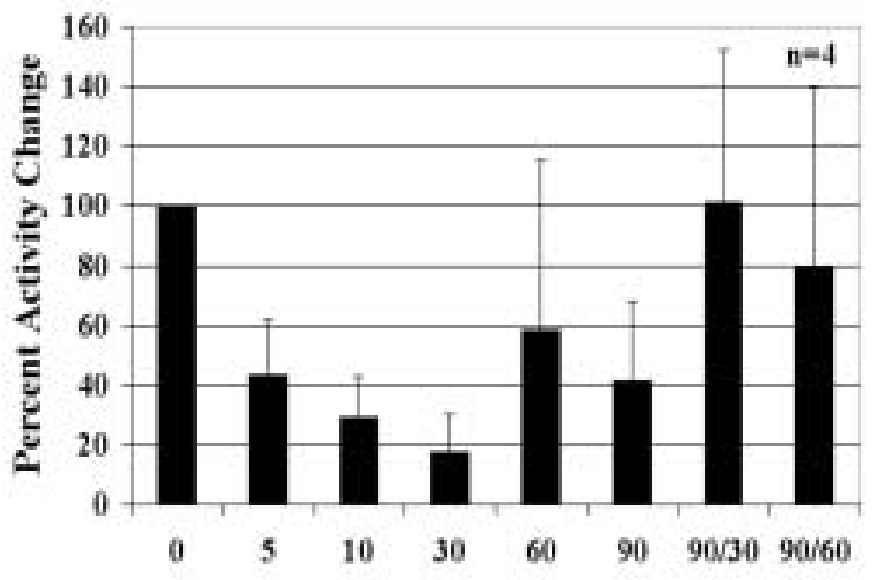

Time of Depletion / Repletion (minutes)

Rho Activity and Ischemia 
Figure 9

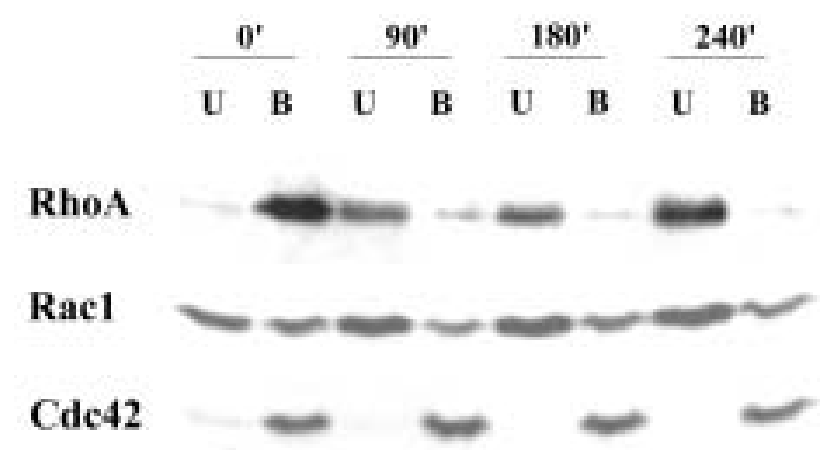

Rho Activity and Ischemia 